\title{
Seleção Dinâmica de Objetos de Aprendizagem Baseados no Padrão SCORM no Ambiente Moodle
}

\author{
João de Amorim Junior, Ricardo Azambuja Silveira \\ Programa de Pós-Graduação em Ciência da Computação (PPGCC) \\ Universidade Federal de Santa Catarina (UFSC) - Florianópolis - SC - Brazil \\ joao.amorimeiate.ufsc.br, ricardo.silveira@ufsc.br
}

\begin{abstract}
This paper presents a model to select Learning Objects (LO) in elearning courses, based on Multiagent paradigm, aiming to facilitate the use of $L O$ in an adaptive way in virtual environments. The proposed framework extends the Intelligent Learning Objects approach through the use of a BDI agent architecture, allowing the communication with the instructional resources that constitute the LO according to the SCORM standard. The agents' reasoning process uses the elements obtained during the interaction between the student and the object, enabling to build enhanced dynamic learning experiences. The LMS Moodle was used to validate this proposed model.
\end{abstract}

Resumo. Este artigo descreve um modelo para seleção de Objetos de Aprendizagem (OA) em cursos a distância, baseado no paradigma de Sistemas Multiagentes, com o intuito de facilitar o uso de OA de forma adaptativa em ambientes virtuais. O framework proposto estende o conceito de Objetos Inteligentes de Aprendizagem, através da utilização de uma arquitetura de agentes BDI, permitindo a comunicação com os recursos instrucionais que constituem o OA de acordo com o padrão SCORM. Os elementos obtidos durante a interação do aluno com o objeto são usados no processo de raciocínio dos agentes, possibilitando o desenvolvimento de experiências de aprendizagem dinâmicas aprimoradas. O LMS Moodle foi usado para validar o modelo proposto.

\section{Introdução}

O Ensino a Distância $(\mathrm{EaD})$ vem contribuindo para a universalização do acesso à educação no Brasil e no mundo [Detoni et al. 2014], sobretudo com a recente evolução da Internet [Pereira et al. 2014]. Diversas instituições já adotaram o e-learning, e novas formas de ensino on-line são cada vez mais presentes, tais como, os cursos massivos (MOOCs) e os ambientes 3D [Allison et al. 2012]. Assim, percebe-se um aumento da demanda por conteúdo $\mathrm{EaD}$ de qualidade. $\mathrm{O}$ uso de diferentes ferramentas computacionais nesta modalidade educacional resulta em melhores experiências de aprendizagem.

Sistemas computacionais de apoio ao ensino devem contribuir para a melhoria de qualidade no processo educativo. Adaptatividade e reuso são importantes aspectos que cooperam para isso. A adaptatividade refere-se à capacidade do ambiente moldar-se às diferentes necessidades e aos estilos de aprendizagem dos alunos. Um sistema adaptável potencializa a compreensão dos conteúdos por parte do aluno, de forma personalizável, levando em conta suas preferências e nível de conhecimento [Mahkameh and Bahreininejad 2011]. O reuso, por sua vez, é um atributo que pode evitar a necessidade 
de se criar novos recursos quando já existam outros materiais similares, previamente produzidos, e que sejam relativos à mesma intenção instrucional [Silva et al. 2012].

Existem algumas ferramentas computacionais que enriquecem o processo de ensino-aprendizagem, tais como: Sistemas Tutores Inteligentes (STI), Sistemas de Gerenciamento de Aprendizagem (LMS, em inglês) e Objetos de Aprendizagem (OA). STI são aplicações desenvolvidas para um domínio educacional específico, cujas arquiteturas geralmente são desenvolvidas $a d-h o c$ e apresentam pouca flexibilidade para reusabilidade e pouca interoperabilidade de recursos [Santos and Jorge 2013], exigindo a reconfiguração do sistema caso sejam necessárias mudanças no domínio. Já os LMS são Ambientes Virtuais de Aprendizagem usados para construção de cursos on-line (ou disponibilização de materiais), permitindo o monitoramento do progresso dos estudantes e o gerenciamento dos dados educacionais [Komlenov et al. 2010]. Por fim, OA são artefatos digitais utilizados com fins educacionais para o aprendizado de conceitos por parte dos alunos, com forte ênfase na reusabilidade [Silva et al. 2012; Santos et al. 2014].

Embora ambientes virtuais baseados em OA favoreçam o reuso de artefatos educacionais e os LMS contribuam para a sua disseminação em larga escala, estes apresentam um grau limitado de adaptação ao aluno, como ocorre nos STI. O tipo de adaptação oferecido por estas ferramentas, de modo geral, precisa ser pré-programado pelo designer instrucional ou professor dentro do recurso ou ambiente, resultando em sistemas que em sua maioria não são dinamicamente adaptáveis [Komlenov et al. 2010].

Este artigo apresenta uma proposta para integrar os três tipos diferentes de paradigmas citados para a concepção de ambientes inteligentes de aprendizagem, utilizando os OA como as unidades instrucionais básicas. Após as etapas iniciais do trabalho, onde foi elaborado um framework baseado em um Sistema Multiagentes para busca e exibição de OA, juntamente com o monitoramento da interação do aluno com o OA [Amorim Jr. et al. 2015], houve a extensão do modelo para permitir aos agentes a comunicação com os recursos do OA segundo o padrão SCORM - Sharable Content Object Reference Model (2004). Com isso, possibilitou-se que elementos do modelo de dados previstos para os OA implementados no padrão fossem usados como insumo no processo de raciocínio dos agentes, permitindo definir dinamicamente a necessidade de utilização de outros OA relacionados ao atual, de acordo com o desempenho do estudante durante a sua interação com este OA. Além disso, foi criado um plug-in para o LMS Moodle, integrando o ambiente de agentes ao LMS, de forma transparente ao aluno.

$\mathrm{O}$ artigo possui a seguinte organização: na seção 2 , os trabalhos correlatos são apresentados; a seção 3 explica a metodologia utilizada e cita detalhes do modelo proposto; a avaliação é descrita na seção 4; por fim, a seção 5 apresenta as conclusões.

\section{Trabalhos Relacionados}

Como ponto de partida desta pesquisa, uma revisão sistemática da literatura foi realizada no portal de periódicos da CAPES/MEC, observando trabalhos existentes na área de sistemas de adaptação de conteúdos educacionais. Posteriormente, realizou-se busca semelhante no sistema de pesquisa dos Anais do SBIE e da RBIE. Como resultado, foram identificados diversos estudos similares a este trabalho. Algumas pesquisas observadas relatam sistemas construídos como uma extensão do LMS, usando diferentes estratégias de adaptação do ambiente, tais como: redes bayesianas [Bachari et al. 2011] e 
data mining [Despotovic et al. 2012]. Há ainda trabalhos não integrados ao LMS, e que usam formas distintas de adaptação da experiência instrucional, segundo o modelo do aluno, ex.: sistemas de recomendação [Vesin et al. 2013; Pereira et al. 2014], regras de produção [Carvalho et al. 2014], lógica fuzzy [Santos et al. 2014] e inteligência de enxames (swarm) [Moura e Fernandes 2012].

Alguns trabalhos analisados agregam o paradigma de Sistemas Multiagentes (SMA) ao LMS resultando em maior adaptação do ambiente, por exemplo: 1) utilização dos dados obtidos da atividade fórum no LMS Moodle (2015) para exibir recursos e outras atividades aos alunos [Alencar and Netto 2011]; 2) ambiente 3D para recomendação de OA baseado no perfil do aprendiz, usando dados do Moodle [Frade et al. 2014]; 3) desenvolvimento de um ambiente inteligente adaptável, baseado em agentes que identificam o perfil do aluno [Giuffra and Silveira 2013]; e 4) SMA apto a selecionar dinamicamente recursos construtivistas no Moodle [Bremgartner et al. 2014].

Com base neste estudo foi possível verificar os principais pontos que os trabalhos apresentam, relacionado a forma como é utilizado o Modelo do Aluno, o conceito de Objeto de Aprendizagem, o acesso aos dados decorrentes da interação do aluno com o ambiente, a integração com LMS e o tipo de adaptatividade obtido. A Tabela 1 exibe a comparação entre as pesquisas correlatas e este trabalho.

Tabela 1. Comparação entre os trabalhos relacionados e o modelo proposto

\begin{tabular}{|c|c|c|c|c|c|}
\hline Trabalho & $\begin{array}{c}\text { Modelo do } \\
\text { Aluno } \\
\end{array}$ & $\begin{array}{c}\text { Padrão } \\
\text { OA }\end{array}$ & $\begin{array}{l}\text { Monitora } \\
\text { Interação }\end{array}$ & $\begin{array}{c}\text { Acessado } \\
\text { no LMS } \\
\end{array}$ & $\begin{array}{l}\text { Âmbito de } \\
\text { Adaptação }\end{array}$ \\
\hline Moura e Fernandes (2012) & Questionário & Não & Não & Não & Ambiente \\
\hline Vesin et al. (2013) & Questionário & SCORM & Não & Não & Ambiente \\
\hline Carvalho et al. (2014) & Questionário & LOM & Não & Não & Ambiente \\
\hline Bachari et al. (2011) & Questionário & LOM & Não & Sim & Ambiente \\
\hline Despotovic et al. (2012) & Questionário & Não & Não & Sim & Ambiente \\
\hline Alencar and Netto (2011) & Fórum & Não & Não & Sim & Ambiente \\
\hline Giuffra and Silveira (2013) & Avaliações & Não & Não & Sim & Ambiente \\
\hline Bremgartner et al. (2014) & $\begin{array}{c}\text { Questionário, } \\
\text { Avaliações e } \\
\text { Frequência }\end{array}$ & Não & Não & Sim & Ambiente \\
\hline Pereira et al. (2014) & Facebook & LOM & Não & Não & Ambiente \\
\hline Frade et al. (2014) & Contexto & SCORM & Não & Não & Ambiente \\
\hline Santos et al. (2014) & Interação & $\begin{array}{c}\text { SCORM } \\
\text { (Estendido) }\end{array}$ & Sim & Não & Ambiente \\
\hline Este trabalho & $\begin{array}{c}\text { Avaliações e } \\
\text { Interação }\end{array}$ & $\begin{array}{c}\text { LOM e } \\
\text { SCORM } \\
\end{array}$ & Sim & Sim & $\begin{array}{c}\text { Objeto de } \\
\text { Aprendizagem }\end{array}$ \\
\hline
\end{tabular}

\section{Metodologia}

Trabalhos anteriores realizados pelo grupo de pesquisa propuseram a junção das tecnologias de OA e SMA. A estratégia, denominada Objetos Inteligentes de Aprendizagem (OIA, em inglês: ILO) [Silveira et al. 2006; Silva et al. 2008; Bavaresco e Silveira 2009], permite que um OA propicie ao aluno experiências educacionais mais adaptáveis, reusáveis e completas, conforme seu perfil cognitivo e desempenho. Um OIA é, na verdade, um agente capaz de exercer o papel de um OA, e de aprender ao interagir com o estudante e com outros OIA, ampliando o potencial de aprendizagem do aluno.

Os metadados do OA são utilizados de modo a permitir que se identifique qual é o tópico educacional relacionado ao objeto. Assim, os OIA (agentes) são capazes de identificar qual o assunto associado com a experiência de aprendizagem exibida ao alu- 
no, e então apresentar informações complementares à sessão anterior (outro OIA), para suprir a falta de conhecimento do estudante no assunto em questão. Tais situações são identificadas no processo de raciocínio dos agentes BDI, a partir dos dados obtidos da interação do aluno com o OA através da comunicação com a API SCORM.

Com base na abordagem ILO descrita, foi projetada, neste novo trabalho, uma evolução no modelo apresentado por Silva et al. (2008) e Bavaresco e Silveira (2009), com o intuito de desenvolver uma extensão àquele modelo, de modo a satisfazer os critérios elencados na Tabela 1 da seção 2. Neste novo modelo, a estrutura do ambiente multiagente foi readequada levando em consideração as novas características dos agentes BDI da versão mais recente (V3) da plataforma JADEX (2015), explorando o conceito de componentes ativos [Pokahr and Braubach 2013]. O processo de raciocínio dos agentes foi aprimorado, de tal forma que outros elementos da especificação IEEE LOM [Carvalho et al. 2014] e do modelo de dados SCORM sejam usados na tomada de decisão referente à exibição ou não de um novo OA ao aluno. Além disso, um plug-in para o Moodle foi desenvolvido permitindo que o Sistema Multiagente resultante seja acoplado ao LMS, de forma que o estudante interaja com o OA diretamente dentro do ambiente do curso, e que o monitoramento da interação lhe pareça transparente.

O objetivo principal do novo modelo de agentes, agora denominado ILOMAS (acrônimo em inglês para Sistema Multiagente de Objetos Inteligentes de Aprendizagem: Intelligent Learning Object Multi-Agent System) é permitir a seleção dinâmica de OA, no contexto do LMS, segundo o desempenho do aluno tanto na interação interna com o OA (elementos SCORM) quanto na externa, realizada dentro do curso através do LMS, sem a necessidade de inclusão de todos os possíveis OA na estrutura do curso.

O modelo proposto não utiliza, portanto, informações relativas ao perfil de aprendizagem do estudante (ex.: textual, visual, interativo [Vesin et al. 2013]). O escopo desta pesquisa considera essencialmente os resultados de desempenho do aluno na experiência de aprendizagem (notas, tempo de interação, sequenciamento e navegação).

A modelagem do sistema foi feita com base na metodologia de engenharia de software orientada à agentes Prometheus [Padgham and Winikoff 2004], utilizando a ferramenta Prometheus Design Tool (2011).

\subsection{Modelo Proposto: ILOMAS - Análise, Projeto e Implementação}

O resultado do trabalho descrito no item anterior levou ao desenvolvimento da estrutura do ILOMAS (Figura 1), que contempla os seguintes agentes:

- LMSAgent - É a representação do LMS, responsável por: (1) receber a requisição do aluno para uma nova experiência de aprendizagem; (2) identificar qual o tema que o estudante deve aprender no momento (objetivo IdentifyLearningExperienceSubject); e (3) passar o controle da interação com o aluno para um novo agente do tipo ILOAgent. Suas crenças advêm de informações da base de dados do LMS, tais como estudantes inscritos, o curso e o assunto que o aluno deve aprender. A comunicação entre este agente e o LMS é feita através de uma API de acesso à banco de dados (ex.: Java JDBC, PHP PDO) ou via Web Services.

- ILOAgent - Exibe a experiência educacional ao aprendiz, isto é, apresenta o OA associado ao assunto definido na crença do agente (obtida da comunicação com 
o LMSAgent). Busca no repositório de objetos por um OA relativo ao tema que o aluno precisa estudar, para então exibi-lo (objetivo SearchLearningObject). Este tipo de agente deve monitorar a interação entre o aluno e o OA (objetivo PlayLearningObject), sendo esta a principal tarefa do SMA. É através dos dados obtidos da interação (os elementos do modelo de dados SCORM, ex.: duração da sessão, nota no questionário, grau de progresso no OA, etc.), e do histórico das interações prévias, que o mecanismo de deliberação do ILOAgent irá definir se o aluno precisa complementar a experiência atual com um objeto extra (se houver demora no acesso ao OA, por exemplo), alterando-se o conteúdo do curso dinamicamente, ou se o aprendiz deve continuar interagindo com o OA atual.

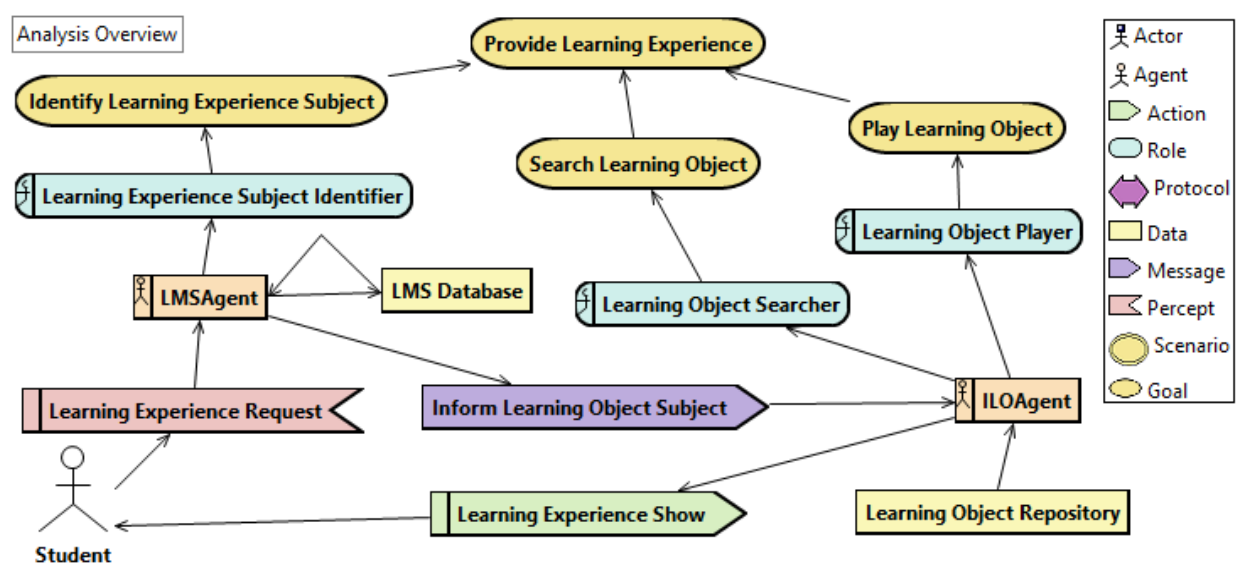

Figura 1. Diagrama de visão geral da análise

O projeto arquitetural do ILOMAS (Figura 2) levou em consideração as características de cursos e-learning disponibilizados em um LMS (como o Moodle), no que se refere à forma de acesso a estes ambientes, que geralmente é feita via web browsers (navegadores). Como resultado, a infraestrutura da plataforma ILOMAS foi modelada levando em consideração requisitos de comunicação entre navegadores (clientes) e servidores web. A interface de interação entre o ambiente de agentes e o ambiente virtual tem como alicerce as tecnologias Java Servlets e JSP, beneficiando-se da estrutura de comunicação de serviços do JADEX BDI V3. A tecnologia de Servlets permite a execução de serviços e classes Java no servidor web, a partir de requisições do navegador.

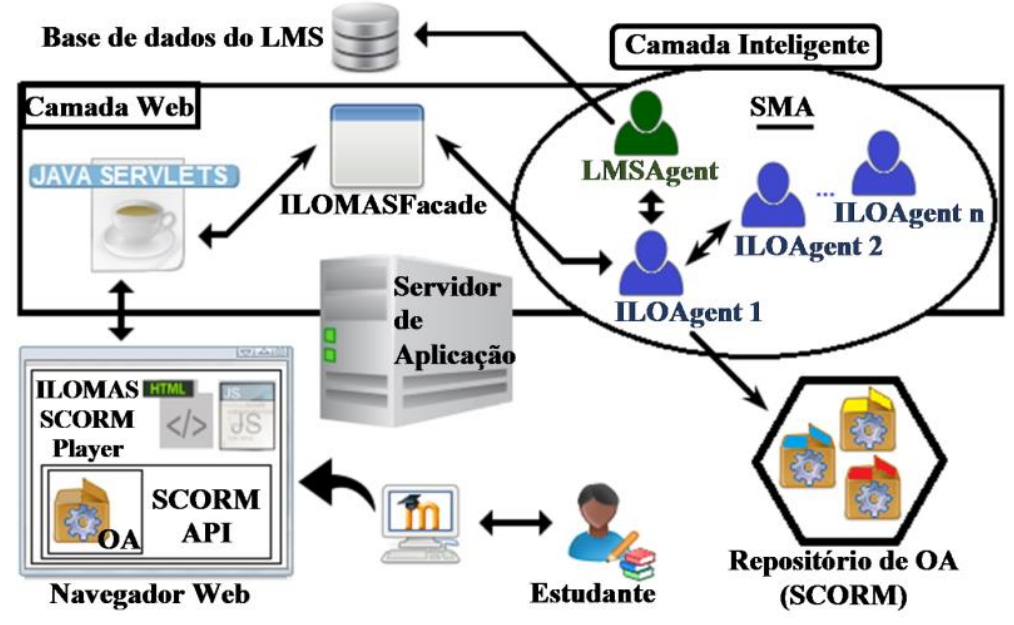

Figura 2. Arquitetura do sistema 
O padrão SCORM define o protocolo de comunicação entre o LMS e o objeto. Por meio deste protocolo, o aprendiz, através do LMS interage com o OA. Desse modo, o estudante visualiza o objeto e controla o sequenciamento de itens que fazem parte do pacote SCORM. Esta comunicação ocorre através de funções JavaScript, a tecnologia mais utilizada mundialmente para desenvolvimento de programação na camada cliente (Browser) na web, adicionando comportamento dinâmico (ex.: AJAX) a conteúdos estáticos (ex.: arquivos HTML) [Morrison 2008]. Para implementar a interface entre o motor SCORM e o ambiente ILOMAS foi necessário desenvolver um player SCORM customizado (baseado em arquivos HTML e JavaScript). Esta estratégia permite que se execute o processamento necessário entre o código SCORM e a camada de agentes.

\subsection{ILOIR: Moodle plug-in}

Para integrar o ambiente ILOMAS ao LMS Moodle de maneira transparente para o aluno, sem que este precise sair do ambiente virtual e acessar outro sistema (evitando possíveis perdas de atenção), foi desenvolvido um plug-in para o LMS, sendo um recurso de interface aos Objetos Inteligentes de Aprendizagem, denominado ILOIR (acrônimo em inglês: Intelligent Learning Object's Interface Resource). Este recurso permite que o professor defina: o assunto (tema) objetivo de aprendizagem daquela unidade instrucional; e o endereço onde a plataforma ILOMAS está disponível (Figura 3). Além disso, o plug-in ILOIR disponibiliza as funcionalidades do sistema ILOMAS ao aluno dentro do Moodle. A extensão desenvolvida acopla a camada web do ILOMAS (player SCORM, contêiner HTML e biblioteca JavaScript) à estrutura do LMS, permitindo que o recurso no Moodle estabeleça comunicação (indireta) com o ambiente dos agentes.

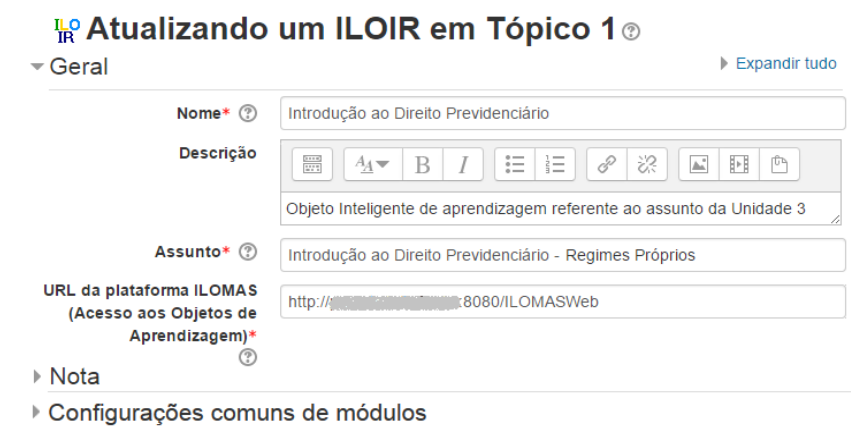

Figura 3. Tela de configuração do plug-in ILOIR

\section{Avaliações}

Os experimentos para avaliação da arquitetura proposta consistem na utilização do modelo para a criação de um curso introdutório de Direito Previdenciário (Dir. Prev.), oferecido por um órgão público de âmbito estadual. O Modelo de avaliação buscou comparar dois tipos de cenários: um de configuração de cursos, na forma convencional e outro de interação de alunos com o recurso ILOIR. Para tanto, foram realizadas comparações entre dois cursos criados no Moodle (Figura 4). O primeiro com três OA do curso de Dir. Prev. (disponíveis no repositório) inseridos isoladamente como recursos do tipo SCORM (nativo no Moodle). O segundo configurado com apenas um recurso do tipo ILOIR (novo plug-in desenvolvido), cujo assunto refere-se ao tema do primeiro objeto SCORM inserido no curso mencionado anteriormente. Com o intuito de facilitar a descrição, daqui em diante o curso com objetos SCORM será chamado "Curso 1" e o curso 
com o recurso ILOIR será denominado "Curso 2". Destaca-se que o intuito desta etapa é avaliar o comportamento adaptativo do sistema, e não sua efetividade educacional.
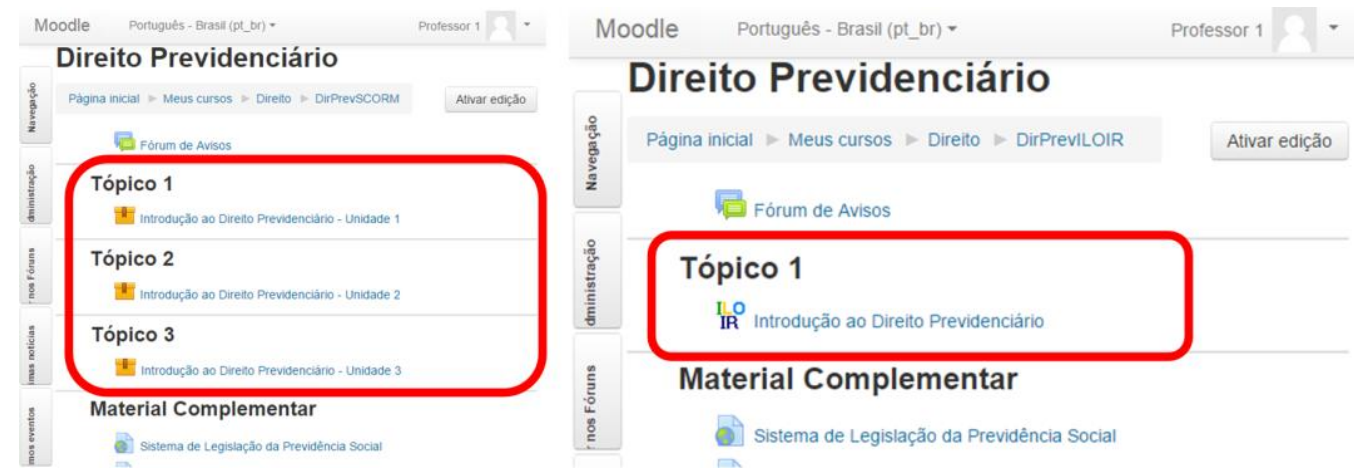

Figura 4. Estrutura do curso com SCORM padrão (esquerda) e ILOIR (direita)

A Tabela 2 compara os dois cursos supracitados configurados no Moodle. Além da redução na quantidade de itens a serem adicionados à estrutura do curso (resultando em um menor tempo de configuração) obtida com o uso do recurso ILOIR, o ponto de maior destaque na comparação refere-se à quantidade de objetos que podem vir a ser utilizados em uma sessão (experiência) de aprendizagem pelo aluno. Enquanto o curso que utiliza os OA explicitamente incluídos possui apenas o objeto adicionado como possibilidade de exibição, o curso que utiliza o plug-in ILOIR (que dá suporte aos Objetos Inteligentes de Aprendizagem do ambiente ILOMAS) permite que de maneira dinâmica vários $\mathrm{OA}$ distintos sejam apresentados ao aluno durante a experiência, desde que estes objetos estejam relacionados ao tema configurado pelo professor no recurso ILOIR. Esta característica oferecida pelo plug-in facilita o reuso de OA.

Tabela 2. Resultados da configuração do curso no LMS

\begin{tabular}{c|c|c|c|c|c} 
& $\begin{array}{c}\text { Recursos } \\
\text { incluídos }\end{array}$ & $\begin{array}{c}\text { OA } \\
\text { incluídos }\end{array}$ & $\begin{array}{c}\text { Tempo de } \\
\text { configuração }\end{array}$ & $\begin{array}{c}\text { Tempo total } \\
\text { de configuração }\end{array}$ & $\begin{array}{c}\text { Quantidade de } \\
\text { OA } \\
\text { disponíveis }\end{array}$ \\
\hline Curso 1 & 3 & 3 & $00: 02: 18$ & $00: 08: 30$ & 1 \\
\hline Curso 2 & 1 & 0 & $00: 00: 58$ & $00: 04: 30$ & $\begin{array}{c}\text { Todos os OA } \\
\text { existentes no } \\
\text { Repositório }\end{array}$
\end{tabular}

Com relação aos cenários de interação de estudantes com o plug-in ILOIR, foram avaliados os acessos de três alunos com graus distintos de entendimento sobre Direito Previdenciário (conceitos: ruim, regular e bom), conforme resultados da Tabela 3.

Tabela 3. Resultados da interação dos estudantes com o recurso ILOIR

\begin{tabular}{c|c|c|c|c|c} 
Estudante & $\begin{array}{c}\text { Conceito } \\
\text { parcial }\end{array}$ & $\begin{array}{c}\text { Quantidade } \\
\text { de assuntos } \\
\text { demonstrados }\end{array}$ & $\begin{array}{c}\text { Tempo de atenção } \\
\text { do aluno }\end{array}$ & $\begin{array}{c}\text { Quantidade de } \\
\text { respostas erradas }\end{array}$ & $\begin{array}{c}\text { Quantidade de } \\
\text { OA } \\
\text { extras (reforço) }\end{array}$ \\
\hline Aluno 1 & Bom & 1 & $00: 09: 46$ & 0 & 0 \\
\hline Aluno 2 & Regular & 2 & $00: 21: 13$ & 3 & 1 \\
\hline Aluno 3 & Ruim & 3 & $00: 34: 19$ & 6 & 3
\end{tabular}

Nota-se que, dependendo do desempenho do aluno (a quantidade de erros no questionário presente no $\mathrm{OA}$, ou a demora em interagir com o objeto), novos OA adicionais são exibidos ao estudante na mesma sessão de aprendizagem. Ocorre, portanto, a adaptação do conteúdo do OA de modo dinâmico, conforme deliberado pelos agentes (camada inteligente do sistema). A Figura 5 exibe o momento em que é identificada e 
sinalizada ao Aluno 3 a necessidade de novo OA, durante a exibição do objeto referente à Unidade 2, devido aos erros em sequência cometidos pelo aprendiz (baixo desempenho). A Figura 6 apresenta a oferta do novo OA referente à Unidade 1 (pré-requisito da Unidade 2) ao Aluno 3, para que este reforce sua compreensão sobre o assunto base.

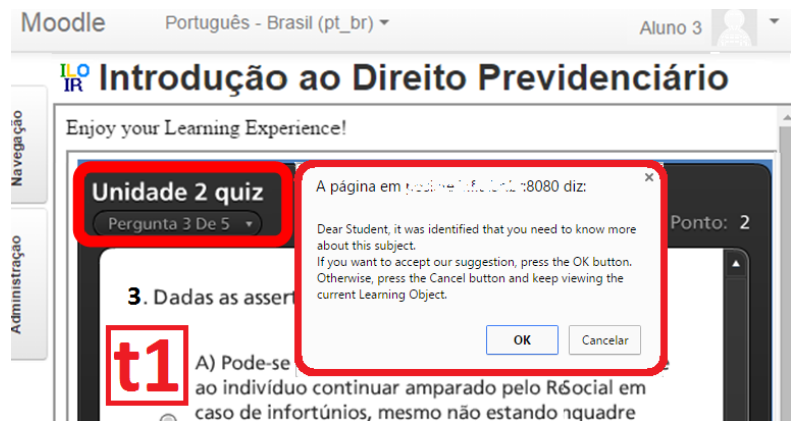

Figura 5. Diagnóstico e sinalização de novo OA ao Aluno 3 (tempo t1)

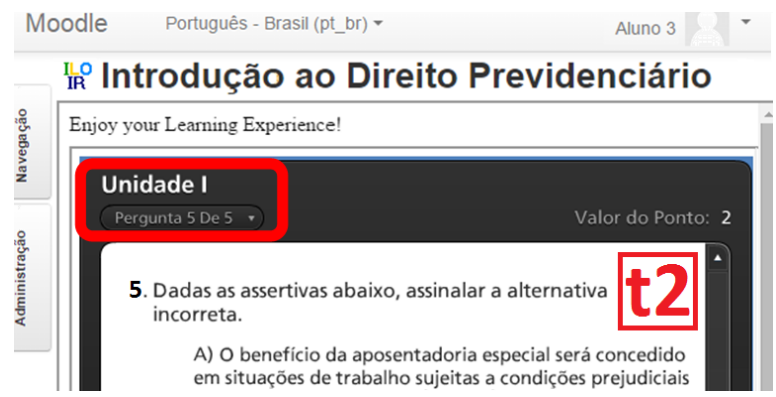

Figura 6. Oferta do novo OA (pré-requisito do OA prévio) ao Aluno 3 (tempo t2)

\section{Considerações Finais}

Este trabalho buscou desenvolver um modelo para construção de experiências educacionais com maior adaptação do conteúdo e favorecimento do reuso, em comparação com outros trabalhos correlatos. A solução descrita permite que OA compatíveis com o padrão SCORM sejam selecionados dinamicamente em cursos oferecidos no LMS Moodle, de acordo com o desempenho do aluno no curso e durante a interação com o OA. O SMA, implementado com base na plataforma JADEX BDI V3, permite que as funcionalidades dos agentes sejam acessadas como serviços, através da camada de integração entre o ambiente ILOMAS (parte inteligente da aplicação) e a camada cliente. A implementação da comunicação entre ILOMAS e a API SCORM possibilita que os dados da interação do aluno com o OA sejam utilizados no processo de deliberação dos agentes. Os testes de avaliação realizados indicaram a viabilidade do modelo proposto.

Como trabalhos futuros, pode-se: integrar o modelo a um sistema de recomendação para indexação e recuperação de OA, como o descrito em Campos et al. (2013), ampliando a lista de OA relativos ao tema definido pelo professor; incluir mecanismos de suporte a ontologias no ambiente ILOMAS, para enriquecer o processo de seleção de novos OA [Allison et al. 2012]; permitir a comunicação do ILOMAS com outros repositórios de OA e outras plataformas de agentes, facilitando a interoperabilidade entre ambientes educacionais distintos [Santos et al. 2006]; promover a compatibilidade do sistema com outros padrões de metadados de OA, tais como Dublin Core e OBAA [Vicari et al. 2010]; bem como elaborar novos cenários de avaliação para o modelo. 
CBIE-LACLO 2015

Anais do XXVI Simpósio Brasileiro de Informática na Educação (SBIE 2015)

\section{Referências}

Allison, C., Miller, A., Oliver, I., Michaelson, R. and Tiropanis, T. (2012) "The Web in education", In: Computer Networks 56, Elsevier, p. 3811-3824.

Alencar, M. and Netto, J. (2011) "Improving Cooperation in Virtual Learning Environments Using Multi-Agent Systems and AIML", In: $41^{\text {st }}$ ASEE/IEEE Frontiers in Education Conference, Session F4C, IEEE, p. 1-6.

Amorim Jr., J., Gelaim, T. and Silveira, R. (2015) "Dynamic e-Learning Content Selection with BDI Agents", In: J. Bajo et al. (Eds.): Highlights on PAAMS. International Workshops of PAAMS 2015, CCIS 524, Springer, p. 299-308.

Bachari, E., Abelwahed, E. and Adnani, M. (2011) "E-Learning personalization based on dynamic learners' preference", In: International Journal of Computer Science \& Information Technology (IJCSIT), Vol 3, No 3. IJCSIT, p. 200-216.

Bavaresco, N. e Silveira, R. (2009) "Proposta de uma arquitetura para construção de Objetos Inteligentes de Aprendizagem baseados em agentes BDI", Anais do XX SBIE.

Bremgartner, V., Netto, J. e Menezes, C. (2014) "Utilizando Agentes e Ontologia de Modelo de Aluno Aberto para Prover Adaptação de Conteúdos Construtivistas em Ambientes Virtuais de Aprendizagem”, Anais do XXV SBIE, p. 1283-1292.

Campos, R., Comarella, R. and Silveira, R. (2013) "Multiagent Based Recommendation System Model for Indexing and Retrieving Learning Objects", In: Highlights on PAAMS, CCIS, Vol. 365, Springer, p. 328-339.

Carvalho, V., Dorça, F., Cattelan, R. e Araújo, R. (2014) “Uma Abordagem para Recomendação Automática e Dinâmica de Objetos de Aprendizagem Baseada em Estilos de Aprendizagem", Anais do XXV SBIE, p. 1188-1197.

Despotovic-Zrakic, M., Markovic, A., Bogdanovic, Z., Barac, D. and Krco, S. (2012) "Providing Adaptivity in Moodle LMS Courses", In: Educational Technology \& Society, 15 (1), IFETS, p. 326-338.

Detoni, D., Araujo, R. e Cechinel, C. (2014) "Predição de Reprovação de Alunos de Educação a Distância Utilizando Contagem de Interações”, Anais do XXV SBIE, p. 896-905.

Frade, R., Neto, F., Lima, R. W., Lima, R. M., Silva, L. e Souza, R. (2014) "Um Ambiente Virtual 3D Multiagente com Recomendação Personalizada de Objetos de Aprendizagem", Anais do XXV SBIE, p. 1068-1077.

Giuffra, P. and Silveira, R. (2013) "A multi-agent system model to integrate Virtual Learning Environments and Intelligent Tutoring Systems", In: International Journal of Interactive Multimedia and Artificial Intelligence, Vol. 2, No. 1, IJIMAI, p. 51-58.

JADEX (2015) Active Components, http:/www.activecomponents.org/

Komlenov, Z., Budimac, Z. and Ivanovic, M. (2010) "Introducing Adaptivity Features to a Regular Learning Management System to Support Creation of Advanced eLessons", In: Informatics in Education, Vol. 9, No. 1, Institute of Mathematics and Informatics, p. 63-80. 
Mahkameh, Y. and Bahreininejad, A. (2011) “A context-aware adaptive learning system using agents", In: Expert Systems with Applications 38, Elsevier, p. 3280-3286.

Moodle (2015) http://www.moodle.org/

Morrison, M. (2008) “Head First JavaScript”, O’Reilly.

Moura, F. e Fernandes, M. (2012) "Uma proposta de um modelo computacional que usa PSO para a escolha de Objetos de Aprendizagem baseado na Espiral de Kolb e nas Inteligências Múltiplas", Anais do XXIII SBIE.

Padgham, L. and Winikoff, M. (2004) "Developing Intelligent Agent Systems - A practical guide", John Wiley \& Sons Ltd.

Pereira, C., Campos, F., Ströele, V., David, J. e Braga, R. (2014) "Extração de Características de Perfil e de Contexto em Redes Sociais para Recomendação de Recursos Educacionais", Anais do XXV SBIE, p. 506-515.

Pokahr, A. and Braubach, L. (2013) "The active components approach for distributed systems development". International Journal of Parallel, Emergent and Distributed Systems, Vol. 28 (4), p. 321-369.

Prometheus Design Tool - Eclipse Plug-in (2011) https://code.google.com/p/pdt-plugin/

Santos, E., Boff, E. and Vicari, R. (2006) "Semantic Web Technologies Applied to Interoperability on an Educational Portal", In: Intelligent Tutoring Systems, LNCS, Vol. 4053, Springer, p. 308-317.

Santos, G. and Jorge, J. (2013) "Interoperable Intelligent Tutoring Systems as Open Educational Resources”, In: IEEE Transactions On Learning Technologies, Vol. 6, No. 3, IEEE CS \& ES, p. 271-282.

Santos, R., Luz, B., Martins, V. e Guimarães, M. (2014) "eTutor: Um Ambiente de Aprendizagem Interativo", Anais do XXV SBIE, p. 476-485.

SCORM (2004) Advanced Distributed Learning, http://www.adlnet.org/scorm

Silva, J., Bavaresco, N. e Silveira, R. (2008) "Projeto e desenvolvimento de um Sistema Multi-agentes para Objetos Inteligentes de Aprendizagem baseado no padrão SCORM", Revista Brasileira de Informática na Educação, Vol. 16, No 01, p. 19-27.

Silva, T., Lima, R., Mesquita, H., Marques, C., Costa, R., Rocha, S. e Medeiros, R. (2012) "OBA-MC: um modelo de Objeto de Aprendizagem centrado no processo de ensino-aprendizagem utilizando o padrão SCORM”, Anais do XXIII SBIE.

Silveira, R., Gomes, E. and Vicari, R. (2006) "Intelligent Learning Objects: An AgentBased Approach of Learning Objects", In: IFIP - International Federation For Information Processing, Vol. 182, Springer, p. 103-110.

Vesin, B., Klasnja-Milicevic, A., Ivanovic, M. and Budimac, Z. (2013) “Applying recommender systems and adaptive hypermedia for e-learning personalization”, In: Computing and Informatics, Vol. 32, Institute of Informatics, p. 629-659.

Vicari, R., Ribeiro, A., Silva, J., Santos, E., Primo, T. and Bez, M. (2010) "Brazilian Proposal for Agent-Based Learning Objects Metadata Standard - OBAA", In: Metadata and Semantic Research, CCIS, Vol. 108, Springer, p. 300-311. 